\title{
Serum and cerebrospinal fluid concentrations of E-selectin in patients with aneurysmal subarachnoid hemorrhage
}

T. Tanriverdi ${ }^{1}$, G.Z. Sanus ${ }^{1}$, M.O. Ulu' ${ }^{1}$, E. Tureci²,

H. Uzun ${ }^{3}$, S. Aydin ${ }^{3}$ and M.Y. Kaynar ${ }^{1}$
${ }^{1}$ Department of Neurosurgery, ${ }^{2}$ Department of Anesthesiology, ${ }^{3}$ Department of Biochemistry, Cerrahpasa Medical Faculty, Istanbul University, Istanbul, Turkey
Correspondence

T. Tanriverdi

P.K: 4, Cerrahpasa

34301, Istanbul

Turkey

Fax: +90-212-414-3427

E-mail: tanerato2000@yahoo.com

Received January 21, 2005 Accepted August 11, 2005

\begin{abstract}
The goal of the present study was to determine concentrations of Eselectin in both cerebrospinal fluid (CSF) and serum of patients with aneurysmal subarachnoid hemorrhage (SAH) and to evaluate the correlation between the clinical parameters and E-selectin levels. Both CSF and serum samples obtained from 12 patients with aneurysmal SAH and 8 patients with hydrocephalus (control group) without any other known central nervous system disease were assayed for Eselectin by quantitative enzyme-linked immunosorbent assay and the results were compared between the two groups. Mean levels of soluble forms of E-selectin within the first 3 days and on the 5th and 7th days of SAH were $4.0 \pm 7.9,2.8 \pm 5.2$, and $3.1 \pm 4.9 \mathrm{ng} / \mathrm{ml}$ in the patient's $\mathrm{CSF}$, and $33.7 \pm 9.2,35.1 \pm 7.0$, and $35.2 \pm 8.7 \mathrm{ng} / \mathrm{ml}$ in serum, respectively. In contrast, mean E-selectin levels were $0.1 \pm 0.2 \mathrm{ng} / \mathrm{ml}$ in CSF and $8.7 \pm 5.0 \mathrm{ng} / \mathrm{ml}$ in serum of control patients. The difference between groups was statistically significant regarding both CSF and serum E-selectin levels $(\mathrm{P}<0.05)$. Thus, we have demonstrated a marked increase of E-selectin concentration in both CSF and serum of patients with aneurysmal SAH compared with control and suggest that blocking the interaction between E-selectin and vascular endothelium may have a beneficial effect on vasospasms.
\end{abstract}

\section{Introduction}

The contribution of vascular endothelium (VE) to inflammation after a pathological condition has been demonstrated and the recruitment of leukocytes from circulating blood and their interaction with endothelium is crucial in inflammatory reactions (1-3). This interaction occurs through a multistep
Key words

- Adhesion molecules

- E-selectin

- Selectin

- Subarachnoid hemorrhage

- Vasospasm process, which includes leukocyte margination, rolling along and firm adhesion to the microvascular endothelium, followed by transmigration through the vessel wall and further migration to extravascular tissue. All phases of the leukocyte recruitment cascade are orchestrated by cell adhesion molecules (CAMs), namely selectins, integrins, and immunoglobulin super-families $(3,4)$. Ap- 
pearance or up-regulation of CAMs has been observed in a variety of central nervous system (CNS) diseases (5-8) and the therapeutic approaches blocking leukocyte-endothelium interactions have provided encouraging results, suggesting that these molecules have a pivotal role (9-13).

Over the last decade, the bulk of the evidence from both experimental and human studies has shown that CAMs may participate in the pathogenesis of vasospasm (VS) after subarachnoid hemorrhage (SAH) following an aneurysm rupture, but few studies have investigated the role of selectins in aneurysmal SAH.

In this report, we focus on the response of one member of the selectin family, E-selectin, that has been proved to participate in the early step of inflammation, namely, rolling of the leukocytes on the VE. We determined the concentrations of E-selectin in both the cerebrospinal fluid (CSF) and serum of patients with aneurysmal SAH and compared them to those of patients with hydrocephalus.

\section{Patients and Methods}

\section{Patients}

Ethical approval for this study was obtained from the Human Investigations Committee at Istanbul University and all patients or the next of kin if the patient was unconscious, provided written informed consent. We studied the patients referred to our neurosurgical unit from January to June 2003 with SAH established by computed tomography (CT). We excluded patients who had any kind of infection at the time of CSF and serum collection, in which CAMs may play a part. The sole inclusion criterion was the admission of the patients to our unit within the first three days of SAH. Demographic and clinical data, i.e., sex, age, initial Glasgow coma scale score, history of chronic illness and smoking, Hunt-Hess (HH) neurological grade, amount of blood within the cisterns determined by Fisher grade on initial CT, initial leukocytosis (white blood cell count more than $11,000 \times 10^{3} / \mathrm{ml}$ ), symptomatic VS, and clinical outcome at 6 months as assessed using the Glasgow outcome scale were recorded on standardized data collection charts. A patient was considered to have symptomatic VS if neurological deterioration (confusion or decreased level of consciousness with focal neurological deficits, such as speech or motor deficits) occurred after day 3 and was managed by the induction of a hyperdynamic state included hypertension, hypervolemia, and hemodilution. If the patients showed clinical signs of symptomatic VS, we performed a CT scan to confirm VS and the scan showed a focal ischemic zone in these patients. In this study, we also tried to determine whether there was a correlation between the levels of E-selectin and the above clinical parameters.

\section{Demographic data of patients and controls}

The study was conducted on 12 patients with aneurysmal SAH and 8 patients with normal pressure hydrocephalus (5 patients) and hydrocephalus secondary to aqueduct stenosis ( 3 patients) without any other known CNS pathology. The patient group included 8 females and 4 males with a mean age of 46.9 years (range: $17-71$ years). The control hydrocephalus group included 4 females and 4 males with a mean age of 44.6 years (range: 14 to 81 years). Among the patients with $\mathrm{SAH}, 4$ had anterior communicating artery aneurysms, 3 had posterior communicating artery aneurysms, 3 had middle cerebral artery aneurysms, and 2 had internal carotid bifurcation aneurysms. Eleven patients underwent surgical clipping, while 1 patient with an anterior communicating artery aneurysm required treatment with endovascular coiling. We performed early surgery $(\leq 48-$ $96 \mathrm{~h}$ post-SAH) in 4 patients with grade II $\mathrm{HH}$ and 4 patients with grade III HH and late 
surgery ( $\geq 10-14$ days post-SAH) in 2 patients with grade IV $\mathrm{HH}$ and 1 patient with grade V HH. One patient with grade II HH underwent endovascular coiling. A summary of the demographic data for the patients with $\mathrm{SAH}$ and the control group is provided in Table 1.

\section{Specimen handling}

Eighty-eight samples (44 each of CSF and serum) were assayed for E-selectin. For each patient, serial blood and CSF samples were collected at the same time within 3 days, and on the 5th and 7 th days of SAH. Blood and CSF samples were collected by venipuncture and lumbar puncture, respectively. In the control hydrocephalus group, blood samples were collected by venipuncture, and CSF samples were obtained during the execution of ventriculo-peritoneal shunting. The samples from the control group were obtained on one occasion. As soon as possible, each 10-ml CSF and blood sample was centrifuged at $10,000 \mathrm{rpm}$ for $15 \mathrm{~min}$ and the supernatant was stored at $-70^{\circ} \mathrm{C}$ until assayed.

\section{E-selectin measurement}

CSF and serum soluble E-selectin (sEselectin) concentrations were measured quantitatively and are reported as $\mathrm{ng} / \mathrm{ml}$ using a commercially available enzyme-linked immunosorbent assay kit (R\&D Systems, Minneapolis, MN, USA, and Abingdon, UK). A dilution of 1:10 was used for E-selectin assays and absorption measurements were carried out at $450 \mathrm{~nm}$ using a microtiter plate reader (Automated Microplate Reader, Mo-

Table 1. Clinical data of the patients with subarachnoid hemorrhage and hydrocephalus controls.

\begin{tabular}{|c|c|c|c|c|c|c|c|c|c|}
\hline & Age/Sex & GCS & $\mathrm{HH}$ & Fisher & Comorbiditya & Leukocytosis & VS & Aneurysm & GOS $^{b}$ \\
\hline \multicolumn{10}{|l|}{ Patient } \\
\hline 1 & $65 / F$ & 10 & IV & 2 & Yes & Yes & Yes & P. Com. A & UF \\
\hline 2 & $67 / F$ & 13 & III & 3 & Yes & Yes & No & A. Com. A & $\mathrm{FV}$ \\
\hline 3 & $58 / \mathrm{M}$ & 13 & III & 3 & Yes & Yes & Yes & ICA & UF \\
\hline 4 & $71 / F$ & 4 & $\mathrm{~V}$ & 3 & Yes & Yes & Yes & MCA & UF \\
\hline 5 & $67 / F$ & 9 & IV & 3 & Yes & No & Yes & P. Com. A & UF \\
\hline 6 & $32 / F$ & 12 & III & 4 & No & No & No & MCA & $\mathrm{FV}$ \\
\hline 7 & $27 / M$ & 14 & II & 2 & No & Yes & No & A. Com. A & FV \\
\hline 8 & $52 / F$ & 14 & ॥ & 2 & No & Yes & No & MCA & $\mathrm{FV}$ \\
\hline 9 & $43 / F$ & 14 & II & 2 & No & No & No & P. Com. A & $\mathrm{FV}$ \\
\hline 10 & 32/M & 13 & III & 3 & No & Yes & No & A. Com. A & UF \\
\hline 11 & $32 / \mathrm{M}$ & 14 & II & 3 & No & Yes & No & A. Com. A & FV \\
\hline 12 & $17 / F$ & 14 & II & 2 & No & Yes & No & ICA & $\mathrm{FV}$ \\
\hline Control & Age/Sex & Diagnosis & & & & & & & \\
\hline 1 & $34 / F$ & $\mathrm{H}^{\mathrm{C}}$ & & & & & & & \\
\hline 2 & $15 / \mathrm{M}$ & $\mathrm{H}^{\mathrm{C}}$ & & & & & & & \\
\hline 3 & $74 / \mathrm{M}$ & $\mathrm{NPH}$ & & & & & & & \\
\hline 4 & $14 / F$ & $\mathrm{H}^{\mathrm{C}}$ & & & & & & & \\
\hline 5 & $45 / F$ & $\mathrm{NPH}$ & & & & & & & \\
\hline 6 & $44 / \mathrm{M}$ & $\mathrm{NPH}$ & & & & & & & \\
\hline 7 & $50 / F$ & $\mathrm{NPH}$ & & & & & & & \\
\hline 8 & $81 / M$ & $\mathrm{NPH}$ & & & & & & & \\
\hline
\end{tabular}

GCS = Glasgow coma scale; HH = Hunt-Hess grade; VS = symptomatic vasospasm; $\mathrm{GOS}=$ Glasgow outcome scale; P. Com. A. = posterior communicating artery; A. Com. A = anterior communicating artery; ICA = internal carotid artery; $\mathrm{MCA}=$ middle cerebral artery; $\mathrm{UF}$ = unfavorable; $\mathrm{FV}=$ favorable; $\mathrm{H}$ = hydrocephalus;

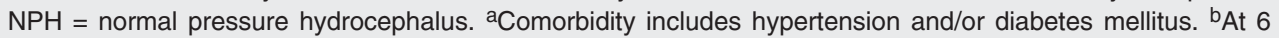
months. CHydrocephalus due to aqueduct stenosis. 
del EC311sx; Biotech Instruments, Winooski, VT, USA). All samples and standards were run in duplicate.

\section{Statistical analysis}

Data were analyzed using the SPSS statistical program (SPSS, Chicago, IL, USA). Statistical analyses were performed using the nonparametric Mann-Whitney U-test. Results are reported as means \pm standard deviation (SD). A probability value of less than 0.05 was considered to be statistically significant.

\section{Results}

Thirty-six CSF and 36 serum samples

Table 2. Cerebrospinal fluid and serum concentrations of E-selectin in 12 patients with subarachnoid hemorrhage and 8 hydrocephalus controls.

\begin{tabular}{lcrrr}
\hline & \multicolumn{4}{c}{ Post-SAH days } \\
\cline { 2 - 5 } & Control & First 3 days & Day 5 & Day 7 \\
\hline E-selectin (CSF) & $0.1 \pm 0.2$ & $4.0 \pm 7.9^{*}$ & $2.8 \pm 5.2^{*}$ & $3.1 \pm 4.9^{*}$ \\
E-selectin (serum) & $8.7 \pm 5.0$ & $33.7 \pm 9.2^{* *}$ & $35.1 \pm 7.0^{\star *}$ & $35.2 \pm 8.7^{* *}$
\end{tabular}

Data are reported as means $\pm \mathrm{SD}$. SAH $=$ subarachnoid hemorrhage; CSF $=$ cerebrospinal fluid. ${ }^{*} \mathrm{P}=0.001$ compared to $\mathrm{CSF}$ of control hydrocephalus group. ${ }^{* *} \mathrm{P}=$ 0.0001 compared to serum of control hydrocephalus group (Mann-Whitney U-test).

Figure 1. Comparison of soluble E-selectin concentrations in the CSF of SAH patients and controls with hydrocephalus and in the serum of SAH patients and controls with hydrocephalus. The squares represent the means \pm SD and the bars denote the range of values $(\mathrm{min} /$ max). The differences in the concentrations of E-selectin between the two groups at the three time points were statistically significant $(P=0.001$ and 0.00001 for CSF and serum, respectively (Mann-Whitney Utest). from the patients with SAH and $8 \mathrm{CSF}$ and 8 serum samples from the control group were obtained for this prospective clinical study. The samples were used for the determination of E-selectin. A summary of the statistical data is provided in Table 2 and Figure 1.

\section{Concentrations of E-selectin in CSF}

Concentrations of E-selectin were markedly different in patients with SAH and the hydrocephalus controls. In the control group, 3 patients had no detectable E-selectin, with a mean concentration of $0.16 \pm 0.21 \mathrm{ng} / \mathrm{ml}$. In contrast, all patients with SAH had quantifiable E-selectin in their CSF. The average values were $4 \pm 7.95,2.84 \pm 5.21$, and $3.10 \pm$ $4.91 \mathrm{ng} / \mathrm{ml}$ within 3 days of SAH, on day 5 , and on day 7 after SAH, respectively. The difference was statistically significant $(\mathrm{P}$ values: $0.002,0.001$, and 0.001 related to days, respectively). Although there appeared to be a trend to a decrease in E-selectin concentrations, the mean levels of E-selectin measured post-SAH at the three time points were not statistically significant when compared with each other $(\mathrm{P}>0.05)$.

\section{Serum E-selectin concentrations}

The mean serum E-selectin concentration for the control group was $8.78 \pm 5.06$ $\mathrm{ng} / \mathrm{ml}$, compared with $33.7 \pm 9.28,35.11 \pm$ 7.07 , and $35.23 \pm 8.75 \mathrm{ng} / \mathrm{ml}$ after 3,5 and 7 days of SAH, respectively. There was a statistically significant difference between control and SAH patients $(\mathrm{P}=0.00001$ for each time point). The patients with SAH showed a tendency to a decrease in serum E-selectin levels during post-SAH days, although the differences were not statistically significant.

\section{Association between sE-selectin concentra-} tions and clinical and demographic variables

Of the 12 SAH patients studied, 4 (pa- 
tients 1, 3, 4, and 5) had symptomatic VS. The E-selectin levels in the CSF of these patients were one order of magnitude higher than the average for all other SAH patients at each time point. The mean $( \pm \mathrm{SD}) \mathrm{sE}$ selectin levels in the CSF of the 4 patients with symptomatic VS measured post-SAH at the three time points were $10.8 \pm 1.7,7.1$ \pm 7.8 , and $7.7 \pm 6.7 \mathrm{ng} / \mathrm{ml}$. In contrast, the mean $( \pm$ SD) levels detected at the same time points in the $8 \mathrm{SAH}$ patients without symptomatic VS were $0.5 \pm 0.2,0.6 \pm 0.2$, and $0.7 \pm 0.4 \mathrm{ng} / \mathrm{ml}$. However, among these patients symptomatic VS had no effect on E-selectin levels $(P>0.05)$, a fact probably due to the small number of patients with SAH included in this study. In addition, no difference in E-selectin levels was observed according to age, sex, initial Glasgow coma scale, $\mathrm{HH}$ grade on presentation, Fisher grade on CT, smoking, co-morbidity, initial leukocytosis, and Glasgow outcome scale at 6 months after SAH. These findings are consistent with the literature but future clinical trials including more patients are needed in order to provide more accurate results.

\section{Discussion}

Recruitment of leukocytes during an inflammatory reaction is a crucial episode, which occurs through a multistep process. The leukocyte-endothelium interaction, the fundamental event of inflammation, is orchestrated by some CAMs found on both leukocytes and endothelial cells and different subsets of these molecules are responsible for the different steps of inflammation $(3,4)$. The capture, rolling along, firm adhesion to, and finally transmigration through the microvascular endothelium of leukocytes are executed step-by-step by the action of CAMs, which are now divided into three families with dissimilar structural architecture: the selectins, the integrins, and some glycoproteins incorporated into the immunoglobulin super-family $(2,4,14-16)$.
For the purpose of this study, we will focus on the selectins.

\section{Selectins and inflammation}

The selectin family of CAMs consists of three members, P-, E-, and L-selectins, all of which mediate rolling of leukocytes along the VE, the early step of the inflammatory process $(17,18)$. P-selectin is found in the granules of endothelium and platelets and comes to the cell surface rapidly in response to several inflammatory stimuli (17). E-selectin, on the other hand, is exclusively expressed on endothelial cells and up-regulated after stimulation by inflammatory cytokines, such as tumor necrosis factor- $\alpha$ and interleukin-1 $(17,18)$. L-selectin is expressed in many subclasses of leukocytes and is rapidly shed from the surface of leukocytes after stimulation (19). It seems that the first contact between leukocytes and VE during inflammation is provided by the induced expression of E- and P-selectin $(1,4,14,15)$. When endothelial cells are activated they first release/express P-selectin and then Eselectin whose adhesion force is sufficiently strong to permit rolling and a reduction in velocity to occur $(1,4,14)$. P-selectin is responsible for the major part of the rolling process of leukocytes, whereas E-selectin mediates slow rolling along the inflamed endothelium $(20,21)$. The interaction between selectin molecules and VE is possible by the action of integrins, a group of macromolecules which are expressed constitutively in leukocytes and in several other types of cells (19). By the end of leukocyte rolling, glycoproteins of the immunoglobulin superfamily cause firm adhesion in order to allow transmigration of leukocytes through the intercellular junction of endothelial cells (22).

Therapeutic targeting of CAMs has provided some beneficial effects in several types of diseases and has disclosed the pivotal role of such molecules in the pathogenesis of 
some pathological conditions of the CNS including VS after SAH (9-13).

\section{Selectins in cerebral ischemia}

Evidence for a pathological role of selectin molecules has been obtained in both animal and human studies. In a primate model of focal ischemia-reperfusion injury, persistent up-regulation of P-selectin in both postcapillary microvascular endothelium and platelets has been shown (23). Wang et al. (24) demonstrated elevation and up-regulation of E-selectin mRNA up to 2 days after middle cerebral artery occlusion in ischemic rat cortex compared with non-ischemic cortex. Up-regulation of E-selectin has been reported in rats to occur in the early period ( 2 to $46 \mathrm{~h}$ ) of reperfusion in rats (25), as well as in middle cerebral artery occlusion and reperfusion injury in non-human primates after $24 \mathrm{~h}$ of reperfusion (26).

Human studies trying to demonstrate the role of selectin molecules in the pathogenesis of cerebral ischemic stroke have provided conflicting data. Shyu et al. (27) found normal levels of E-selectin within $24 \mathrm{~h}$ of ischemic stroke in adults. A fall in E-selectin over 5 days in ischemic stroke patients has also been reported (28). In contrast, significant elevations of P- and E-selectin molecules in the acute stage of ischemic stroke have been demonstrated (29-31). Taken together, these findings and encouraging results of studies with different therapeutic options targeting the interaction between selectins and VE strongly support the notion that selectins may play an important role in the acute stages of cerebral ischemia.

\section{Selectins in subarachnoid hemorrhage}

It has been demonstrated that activation of VE of large cerebral arteries in the subarachnoid space is initiated once the blood contacts the outermost layer of the vessel after SAH (32), leading to a cascade of changes in both the morphology and vasomotor regulation of the exposed vessel, a process that often leads to the clinical condition recognized as VS, with peaks occurring on the 7 th day of SAH $(12,33,34)$. The role of CAMs, principally selectins, in $\mathrm{SAH}$ is less clear and few studies have investigated the changes in selectin molecules in aneurysmal SAH $(35,36)$. Polin et al. (35) showed marked elevation of soluble forms of $E$ selectin in the CSF of 17 patients with ruptured SAH compared with levels in the CSF of 16 controls, patients with unruptured aneurysms, and patients tested months after the occurrence of SAH. In addition, 3 patients with angiographically demonstrated VS showed higher levels of E-selectin than the average for all other SAH patients. More recently, in a prospective study, Nissen et al. (36) compared the serum concentrations of three selectin molecules in patients with and without delayed ischemic neurological deficit (DIND) after grade 1 or $2 \mathrm{SAH}$. The Eselectin levels were similar for the two groups. On the other hand, P-selectin concentration was significantly higher in patients with DIND compared with patients without DIND. By contrast, L-selectin concentration was lower in patients with DIND. The authors concluded that $\mathrm{P}$ - and L-selectin might be involved in the pathophysiology of DIND after aneurysmal SAH.

Ours is the first study in which E-selectin concentrations were evaluated concomitantly in both the CSF and serum of patients after aneurysmal SAH and the small series of patients examined here showed a statistically significant elevation in E-selectin levels in both CSF and serum after aneurysmal SAH compared with controls. The concentrations at three time points after $\mathrm{SAH}$ were higher than control in both CSF and serum, suggesting that $\mathrm{VE}$ is active in the early period of SAH. We may speculate that early treatment targeting the blockage of interaction between E-selectin and VS may reduce inflammation in the early period and prevent 
VS, which is typically possible between 4 and 14 days after the onset of SAH. This may provide an opportunity for prophylactic anticipatory therapy. Our findings are consistent with those in which serum selectin molecules were detected in human stroke, but not with the study by Nissen et al. (36).

There was no statistically significant correlation between E-selectin concentrations and the clinical profile of the patients, consistent with previous human SAH studies related to E-selectin $(35,36)$. Nevertheless, we think that this may be due to the small number of patients studied here.
Our findings suggest that there may be an association between elevated sE-selectin levels in the CSF and serum of patients with aneurysmal SAH and the relevance of our findings for the treatment of VS in SAH remains to be investigated. The importance of our findings is that they suggest that blockage of the E-selectin pathway may be of potential therapeutic benefit to these patients. Additional clinical studies involving a larger number of patients are required in order to determine whether there is a correlation between VS and the elevation of E-selectin molecules.

\section{References}

1. Imhof BA \& Dunon D (1995). Leukocyte migration and adhesion. Advances in Immunology, 58: 345-416.

2. Luscinskas FW \& Gimbrone Jr MA (1996). Endothelial-dependent mechanisms in chronic inflammatory leukocyte recruitment. Annual Review of Medicine, 47: 413-421.

3. Muller WA (2002). Leukocyte-endothelial cell interactions in the inflammatory response. Laboratory Investigation, 82: 521-533.

4. Carlos TM \& Harlan JM (1994). Leukocyte-endothelial adhesion molecules. Blood, 84: 2068-2101.

5. Buhrer C, Herold R, Stibenz D et al. (1996). Cerebrospinal fluid soluble L-selectin ( $\mathrm{SCD} 62 \mathrm{~L}$ ) in meningoencephalitis. Archives of Disease in Childhood, 74: 288-292.

6. Jander S, Heidenreich F \& Stoll G (1993). Serum and CSF levels of soluble intracellular adhesion molecule-1 (ICAM-1) in inflammatory neurological diseases. Neurology, 43: 1809-1813.

7. Rossler K, Neuchrist C, Kitz K et al. (1992). Expression of leucocyte adhesion molecules at the human blood-barrier (BBB). Journal of Neuroscience Research, 31: 365-374.

8. Tsukada N, Matsuda M, Miyagi K et al. (1993). Increased levels of intercellular adhesion molecule-1 (ICAM-1) and tumor necrosis factor receptor in the cerebrospinal fluid of patients with multiple sclerosis. Neurology, 43: 2679-2682.

9. Aydt E \& Wolf G (2002-3). Development of synthetic pan-selectin antagonists: a new treatment strategy for chronic inflammation in asthma. Pathobiology, 70: 297-301.

10. Bavbek M, Polin R, Kwan A et al. (1998). Monoclonal antibodies against ICAM-1 and CD18 attenuate cerebral vasospasm after experimental subarachnoid haemorrhage in rabbits. Stroke, 29: 19301936.

11. Clark WM, Madden KP \& Rothlein R (1991). Reduction of central nervous system ischaemic injury by monoclonal antibody to intercellular adhesion molecule. Journal of Neurosurgery, 75: 623-627.

12. Oshiro EM, Hoffman PA, Dietsch GN et al. (1997). Inhibition of experimental vasospasm with anti-intercellular adhesion molecule1 monoclonal antibody in rats. Stroke, 28: 2031-2038.

13. Romano SJ \& Slee DH (2001). Targeting selectins for the treatment of respiratory diseases. Current Opinion in Investigational Drugs, 2: 907-913.

14. Kansas GS (1996). Selectins and their ligands: current concepts and controversies. Blood, 88: 3259-3287.

15. Springer TA (1994). Traffic signals for lymphocyte recirculation and leukocyte emigration: the multistep paradigm. Cell, 76: 301-314.

16. Zimmerman GA, Prescott SM \& McIntyre TM (1992). Endothelial cell interactions with granulocytes: tethering and signalling molecules. Immunology Today, 13: 93-100.

17. Ley K (2001). Functions of selectins. Results and Problems in Cell Differentiaton, 33: 177-200.

18. Vestweber D \& Blanks JE (1999). Mechanisms that regulate the function of the selectins and their ligands. Physiological Reviews, 79: $181-213$.

19. Takagi J \& Springer TA (2002). Integrin activation and structural rearrangement. Immunological Reviews, 186: 141-163.

20. Xia L, Sperandia M, Yago T et al. (2001). P-selectin glycoprotein ligand-1-deficient mice have impaired leukocyte tethering to E-selectin under flow. Journal of Clinical Investigation, 109: 939-950.

21. Yang J, Hirata T, Croce K et al. (1999). Targeted gene disruption demonstrates that $\mathrm{P}$-selectin glycoprotein ligand 1 (PSGL-1) is required for P-selectin-mediated but not E-selectin-mediated neutrophil rolling and migration. Journal of Experimental Medicine, 190: 1769-1782.

22. Hogg N, Henderson R, Leitinger B et al. (2002). Mechanisms contributing to the activity of integrins on leukocytes. Immunological Reviews, 186: 164-171.

23. Pitzalis C, Pipitone N \& Perreti M (2002). Regulation of leukocyteendothelial interactions by glucocorticoids. Annals of the New York Academy of Sciences, 966: 108-118.

24. Wang X, Yue TL, Barone FC et al. (1995). Demonstration of increased endothelial-leukocyte adhesion molecule-1 mRNA expression in rat ischemic cortex. Stroke, 26: 1665-1668.

25. Slee D, Romano SJ, Yu J et al. (2001). Development of potent noncarbohydrate imidazole-based small molecule selectin inhibitors with anti-inflammatory activity. Journal of Medicinal Chemistry, 44: 2094- 
2107.

26. Haring HP, Berg EL, Tsurushita N et al. (1996). E-selectin appears in non-ischemic tissue during experimental focal cerebral ischemia. Stroke, 27: 1386-1391.

27. Shyu KG, Chang H \& Linn CC (1997). Serum levels of intercellular adhesion molecule-1 and E-selectin in patients with acute ischaemic stroke. Journal of Neurology, 244: 90-93.

28. Bitsch A, Klene W, Murtada L et al. (1998). A longitudinal prospective study of soluble adhesion molecules in acute stroke. Stroke, 29: 2129-2135.

29. Fassbender K, Mossner R, Motsch L et al. (1995). Circulating selectin- and immunoglobulin-type adhesion molecules in acute ischemic stroke. Stroke, 26: 1361-1364.

30. Frijins CJM, Kappelle LJ, van Gijin J et al. (1997). Soluble adhesion molecules reflect endothelial cell activation in ischemic stroke and in carotid atherosclerosis. Stroke, 28: 2214-2218.

31. Wu G, Li F, Li P et al. (1993). Detection of plasma alpha-granule membrane protein GMP-140 using radiolabelled monoclonal antibodies in thrombotic disease. Haemostasis, 23: 121-128.
32. Sonobe M \& Suzuki J (1978). Vasospasmogenic substance produced following subarachnoid hemorrhage, and its fate. Acta Neurochirurgica, 44: 97-106.

33. Handa Y, Kubota T, Kaneko M et al. (1995). Expression of intercellular adhesion molecule 1 [ICAM-1] on the cerebral artery following subarachnoid haemorrhage in rats. Acta Neurochirurgica, 132: 9297.

34. Sills A, Clatterbuck RE, Thompson RC et al. (1997). Endothelial cell expression of intercellular adhesion molecule 1 in experimental posthaemorrhagic vasospasm. Neurosurgery, 41: 453-460.

35. Polin RS, Bavbek M, Shaffrey ME et al. (1998). Detection of soluble E-selectin, ICAM-1, VCAM-1, and L-selectin in the cerebrospinal fluid of patients after subarachnoid hemorrhage. Journal of Neurosurgery, 89: 559-567.

36. Nissen JJ, Mantle D, Gregson B et al. (2001). Serum concentration of adhesion molecules in patients with delayed ischaemic neurological deficit after aneurysmal subarachnoid haemorrhage: the immunoglobulin and selectin superfamilies. Journal of Neurology, Neurosurgery and Psychiatry, 71: 329-333. 